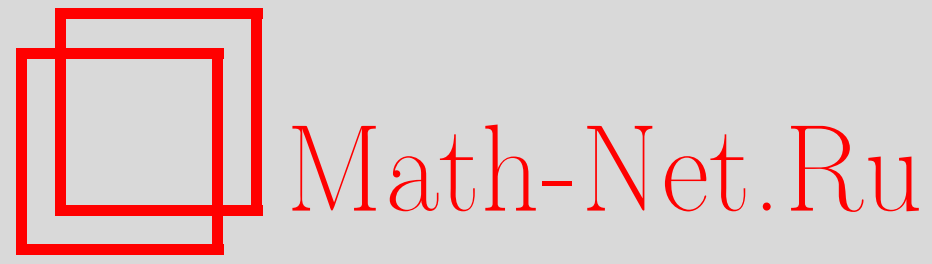

Общероссийский математический портал

Р. О. Гринив, Т. А. Мельник, О сингулярном функционале Рэлея, Матем. заметки, 1996, том 60, выпуск 1, 130-134

DOI: https://doi.org/10.4213/mzm1810 
Использование Общероссийского математического портала MathNet.Ru подразумевает, что вы прочитали и согласны с пользовательским соглашением

http://www . mathnet.ru/rus/agreement

Параметры загрузки:

IP : 3.85 .183 .62

26 апреля 2023 г., 18:09:09

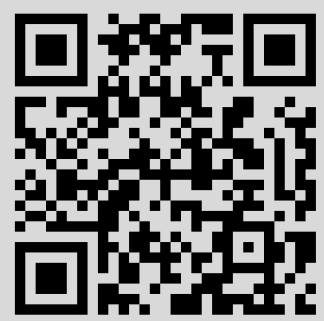




\section{О СИНГУЛЯРНОМ ФУНКЦИОНАЛЕ РЭЛЕЯ}

\section{Р.О. Гринив, Т. А. Мельник}

Настоящая работа посвящена исследованию спектральных свойств операторфункции $(\mathrm{O} \Phi)$

$$
L(\lambda)=A(\lambda)-I, \quad \lambda \in(a, b) \subset \mathbb{R},
$$

где $A(\lambda)$ - непрерьвная самосопряженная компактная ОФ в гилбертовом пространстве $\mathscr{H}$ со скалярным произведением $(*, *), I$ - единичный оператор в $\mathscr{H}$. Кроме этого предполагаем, что выполнено следующее условие:

для любого ненулевого вектора $u \in \mathscr{H}$ функция

$$
f_{u}(\lambda):=(L(\lambda) u, u), \quad \lambda \in(a, b),
$$

возрастает в некоторой окрестности каждого своего нуля, если таковые существуют.

Понятно, что при таких условиях функция $f_{u}$ не может иметь более одного нуля (возможно, кратного).

Спектральные свойства самосопряженных $О \Phi$ со свойством (2) исследовались с помощью функционала Рэлея в [1]-[3]. Отметим, однако, что функционал Рэлея определялся в этих работах при следующих предположениях: во-первых, для каждого ненулевого $u \in \mathscr{H}$ функция $f_{u}$ имеет нуль $p(u) \in(a, b)$; во-вторых, либо ОФ $L(\lambda)$ непрерывно дифференцируема и нуль $p(u)$ функции $f_{u}$ простой $[1]$, либо функционал $p$ ограничен, т.е. $\operatorname{supp} p \Subset(a, b)[2],[3]$.

Работа первого автора выполнена при поддержке Международного научного фонда, грант NCI300. 
Поводом для рассмотрения ОФ вида (1)-(2) послужили работы [4], [5], в которых исследовалось асимптотическое поведение при $\varepsilon \rightarrow 0$ спектра задачи Неймана в густых сингулярно вырождающихся соединениях, зависящих от малого параметpa $\varepsilon$. В этих работах было показано, что предельная $(\varepsilon=0)$ спектральная задача эквивалентна спектральной задаче для ОФ

$$
S(\lambda)=\left(\lambda^{2}+1\right) A+\lambda \operatorname{tg} \lambda B-I, \quad \lambda \in(\pi n-\pi / 2, \pi n+\pi / 2), \quad n \in \mathbb{N},
$$

где $A$ и $B$ - самосопряженные компактные операторы в $\mathscr{H}, A>0, B \geqslant 0$.

К ОФ вида (1) можно также свести квадратичный пучок операторов

$$
T(\lambda)=\lambda^{2} I+\lambda(\alpha A+B)+A+C,
$$

рассматриваемый в работе [6]; здесь $A=A^{*} \gg 0$, обратный оператор $A^{-1}$ компактен, операторы $B$ и $C$ симметричны и $A$-компактны. Спектры пучков $T(\lambda)$ и

$$
\begin{aligned}
T_{1}(\lambda) & =-(1+\lambda \alpha)^{-1} A^{-1 / 2} T(\lambda) A^{-1 / 2} \\
& =-(1+\lambda \alpha)^{-1}\left[\lambda^{2} A^{-1}+\lambda A^{-1 / 2} B A^{-1 / 2}+A^{-1 / 2} C A^{-1 / 2}\right]-I
\end{aligned}
$$

совпадают, а пучок $T_{1}(\lambda)$ в интервале $\left(a_{3},-1 / \alpha\right)$ удовлетворяет условиям (1)-(2).

Легко видеть, что к ОФ $S(\lambda)$ и $T(\lambda)$ не могут быть применены методы работ [1]-[3], а метод, предложенный в работах [4], [5], [7], не является общим для ОФ вида (1)-(2).

В настоящей статье построен сингулярный функционал Рэлея, с помощью которого установлены следующие результаты:

1) спектр ОФ $L(\lambda)$ на $(a, b)$ дискретен;

2) единственно возможной точкой накопления собственных значений является точка $\lambda=b$; приведен критерий накопления;

3) для собственных значений доказаны вариационные принципы.

1. Исследование спектра ОФ $L(\lambda)$. Напомним, что точка $\mu_{0} \in(a, b)$ является регулярной точкой ОФ $L(\lambda)$, если оператор $L\left(\mu_{0}\right)$ ограниченно обратим. Множество всех регулярных точек ОФ $L(\lambda)$ обозначим через $\rho(L)$. Cnектром $\sigma(L)$ ОФ $L$ в интервале $(a, b)$ называется множество $\sigma(L)=(a, b) \backslash \rho(L)$. Число $\lambda_{0} \in \sigma(L)$ называется собственным значением (СЗ) (геометрической) кратности $n=n\left(\lambda_{0}\right)(n>0)$, если $\operatorname{dim} \operatorname{Ker} L\left(\lambda_{0}\right)=n$; при этом любой ненулевой вектор $u_{0} \in \operatorname{Ker} L\left(\lambda_{0}\right)$ назьвается собственным вектором (CB), отвечающим СЗ $\lambda_{0}$.

Пусть $\lambda_{0} \in \sigma(L)$, т.е. оператор $A\left(\lambda_{0}\right)-I$ не имеет ограниченного обратного. Так как оператор $A\left(\lambda_{0}\right)$ компактен, то число 1 является его конечнократньм СЗ. Следовательно, спектр $\sigma(L)$ состоит из СЗ конечной кратности.

Определим сингулярный функиионал Рэлея $p: \mathscr{H} \backslash\{0\} \rightarrow[a, b]$ следующим образом:

$$
p(u)= \begin{cases}a, & \text { если } f_{u}(\lambda)>0 \forall \lambda \in(a, b) ; \\ \alpha, & \text { если } f_{u}(\alpha)=0 ; \\ b, & \text { если } f_{u}(\lambda)<0 \forall \lambda \in(a, b) .\end{cases}
$$

Очевидно, что для $\lambda \in(a, b)$ справедливы соотношения

$$
\begin{aligned}
& p(u)>\lambda \Longleftrightarrow(L(\lambda) u, u)=f_{u}(\lambda)<0 ; \\
& p(u)=\lambda \Longleftrightarrow(L(\lambda) u, u)=f_{u}(\lambda)=0 ; \\
& p(u)<\lambda \Longleftrightarrow(L(\lambda) u, u)=f_{u}(\lambda)>0,
\end{aligned}
$$

из которых следует непрерьвность функционала $p$. 
ПРЕДЛОЖЕНИЕ 1 (см. [2]). Пусть $\left\{\lambda_{k}\right\}_{k=1}^{n}(n \in \mathbb{N}$ uлu $n=\infty)-$ cuстем $a$ попарно различных СЗ ОФ $L(\lambda)$. Тогда

1) система соответствующих $C B\left\{u_{k}\right\}$ линейно независима;

2) для произвольного ненулевого вектора $g$ из замкнутой линейной оболочки системы векторов $\left\{u_{k}\right\}$ имеем

$$
\inf \left\{\lambda_{k}\right\} \leqslant p(g) \leqslant \sup \left\{\lambda_{k}\right\}
$$

Обозначим через $N(\alpha, \beta)$ количество СЗ ОФ $L(\lambda)$ в интервале $(\alpha, \beta)$ с учетом кратности, а через $\pi(\mu)$ - количество положительных СЗ оператора $L(\mu)$ с учетом кратности. Очевидно, что $\pi(\mu)$ - конечная неубывающая функция.

Теорема 1. Пусть $\alpha$ и $\beta$-регулярные точки ОФ $L(\lambda)$. Тогда

$$
N(\alpha, \beta)=\pi(\beta)-\pi(\alpha) .
$$

ДоказАтЕльство. Сначала докажем, что $N(\alpha, \beta) \leqslant \pi(\beta)-\pi(\alpha)$. Для этого достаточно показать, что при прохождении аргумента $\lambda$ через С3 $\lambda_{0}$ значение функции $\pi(\lambda)$ увеличивается не менее, чем на $n\left(\lambda_{0}\right)=\operatorname{dim} \operatorname{Ker} L\left(\lambda_{0}\right)$.

Зафиксируем достаточно малое $\varepsilon>0$ ипусть $\mathscr{H}=\mathscr{H}^{+} \oplus \mathscr{H}^{-}$- разбиение пространства $\mathscr{H}$ в ортогональную сумму $L\left(\lambda_{0}-\varepsilon\right)$-положительного и $L\left(\lambda_{0}-\varepsilon\right)$-неположительного инвариантных подпространств, порожденное спектральным разложением единицы оператора $L\left(\lambda_{0}-\varepsilon\right)$. Тогда $\operatorname{dim} \mathscr{H}^{+}=\pi\left(\lambda_{0}-\varepsilon\right)$ и $\forall u \in \mathscr{H}^{+} \backslash\{0\}$ имеем $\left(L\left(\lambda_{0}\right) u, u\right)>0$. Подпространства $\mathscr{H}^{+}$и $\operatorname{Ker} L\left(\lambda_{0}\right)$ линейно независимы, ибо $p(x)<\lambda_{0} \forall x \in \mathscr{H}^{+} \backslash\{0\}$, тогда как $p(y)=\lambda_{0} \forall y \in \operatorname{Ker} L\left(\lambda_{0}\right) \backslash\{0\}$. Более того, линеал $\mathscr{L}=\mathscr{H}^{+} \dot{+} \operatorname{Ker} L\left(\lambda_{0}\right)$ является $L\left(\lambda_{0}\right)$-неотрицательным, так как для $u=x+y, x \in \mathscr{H}^{+}, y \in \operatorname{Ker} L\left(\lambda_{0}\right)$, имеем $\left(L\left(\lambda_{0}\right) u, u\right)=\left(L\left(\lambda_{0}\right) x, x\right) \geqslant 0$. Поэтому $\left(L\left(\lambda_{0}+\varepsilon\right) u, u\right)>0 \forall u \in \mathscr{L} \backslash\{0\}$, и, следовательно, $\pi\left(\lambda_{0}+\varepsilon\right) \geqslant \operatorname{dim} \mathscr{L}=$ $\pi\left(\lambda_{0}-\varepsilon\right)+n\left(\lambda_{0}\right)$.

Докажем обратное неравенство. Заметим, что все неотрицательные СЗ оператора $L(\lambda)$ зависят от $\lambda$ непрерьвно. В частности, функция $\pi(\lambda)$ имеет скачок в точке $\lambda_{0}$ тогда и только тогда, когда нуль является СЗ оператора $L\left(\lambda_{0}\right)$, т.е. $\lambda_{0}-\mathrm{C} 3 \mathrm{O} \Phi$ $L(\lambda)$. Таким образом, $N(\alpha, \beta) \geqslant \pi(\beta)-\pi(\alpha)$, и теорема доказана.

ЗАмЕч АниЕ 1 . В случае, когда по крайней мере одна из точек $\alpha$ или $\beta$ принадлежит спектру $\sigma(L)$, верно равенство $N(\alpha, \beta)=\pi(\beta)-\pi(\alpha+0)$.

СлЕДСтвИЕ. Любой отрезок из интервала $(a, b)$ содержит не более чем конечное число СЗ ОФ $L(\lambda)$, m.е. спектр $О \Phi L(\lambda)$ в интервале $(a, b)$ дискретен. Точкой накопления СЗ ОФ $L(\lambda)$ мохет служить лишь точка $\lambda=b$, что происходит в том и только том случае, когда $\pi(\lambda) \rightarrow \infty$ при $\lambda \rightarrow b-0$.

ЗАмЕч АниЕ 2 . Легко видеть, что все доказанные утверждения останутся справедливыми, если вместо компактности ОФ $A(\lambda)$ в $(1)$ потребовать, чтобы для всех $\lambda$ из интервала $(a, b)$ существенный спектр оператора $L(\lambda)$ не содержал неотрицательных точек и $\pi(\lambda)<\infty$.

2. Вариационные принципы. Докажем теперь для $О \Phi L(\lambda)$ аналоги классических вариационных принципов. Расположим СЗ ОФ $L(\lambda)$ в неубывающем порядке с учетом их кратностей:

$$
a<\lambda_{1} \leqslant \lambda_{2} \leqslant \ldots \leqslant \lambda_{n} \leqslant \cdots .
$$


Положим $\pi\left(\lambda_{1}\right)=m$ и пусть $\mathscr{H}=\mathscr{H}_{k}^{+} \oplus \mathscr{H}_{k}^{-}-$разбиение пространства $\mathscr{H}$ в ортогональную сумму $L\left(\lambda_{k}\right)$-положительного и $L\left(\lambda_{k}\right)$-неположительного $L\left(\lambda_{k}\right)$-инвариантных подпространств. Тогда $\operatorname{dim} \mathscr{H}_{k}^{+}=\pi\left(\lambda_{k}\right)=m+k^{\prime}$, где $k^{\prime}<k$. Обозначим через $M_{m+k}$ подпространство размерности $m+k$, натянутое на $\mathscr{H}_{k}^{+}$и попарно ортогональные СВ $u_{k^{\prime}+1}, \ldots, u_{k}$, отвечающие С $3 \lambda_{k^{\prime}+1}=\cdots=\lambda_{k}$. Понятно, что для любого ненулевого вектора $u \in M_{m+k}$ (соответственно $u \perp M_{m+k}$ ) выполнено неравенство $p(u) \leqslant \lambda_{k}$ (соответственно $\left.p(u) \geqslant \lambda_{k}\right)$. Пусть также $\mathbb{E}_{n}$ $\left(\right.$ соответственно $\mathbb{E}^{n}$ ) есть множество всех подпространств пространства $\mathscr{H}$ размерности $n$ (соответственно коразмерности $n$ ).

Предварительно докажем следующее утверждение.

ПРЕДЛОЖЕНИЕ 2. Пусть Е - подпространство Н̈. Тогда функиионал Рэлея $р$ достигает на Е своего минимума.

ДокАЗАТЕЛЬСтво. Положим $\alpha_{0}:=\inf _{0 \neq u \in E} p(u)$. Случай $\alpha_{0}=b$ тривиален, поэтому пусть $\alpha_{0}<b$ и последовательность векторов $\left\{u_{n}\right\} \subset E$ такова, что $\left\|u_{n}\right\|=1$ и $p\left(u_{n}\right) \rightarrow \alpha_{0}$. Можно считать, что $u_{n}$ слабо сходятся к $u_{0} \in E$, $\left\|u_{0}\right\| \leqslant 1$. Пусть $\alpha>\alpha_{0}$, тогда ввиду компактности оператора $A(\alpha)$ для достаточно больших $n$ имеем

$$
\begin{aligned}
0 \leqslant\left(L(\alpha) u_{n}, u_{n}\right) & =\left(A(\alpha) u_{n}, u_{n}\right)-\left(u_{n}, u_{n}\right) \rightarrow\left(A(\alpha) u_{0}, u_{0}\right)-1 \\
& \leqslant\left(A(\alpha) u_{0}, u_{0}\right)-\left(u_{0}, u_{0}\right)=\left(L(\alpha) u_{0}, u_{0}\right) .
\end{aligned}
$$

Следовательно, $u_{0} \neq 0$ и $p\left(u_{0}\right) \leqslant \alpha$, откуда ввиду произвольности $\alpha>\alpha_{0}$ получаем $p\left(u_{0}\right)=\alpha_{0}$.

ТЕОРема 2. Для CЗ $\lambda_{1}, \lambda_{2}, \ldots, \lambda_{n}, \ldots$ ОФ L справедлив вариационный принцип Пуанкаре-Ритца:

$$
\lambda_{n}=\max _{E \in \mathbb{E}^{n+m-1}} \min _{0 \neq u \in E} p(u) .
$$

ДоказАтЕльство. Если $E \in \mathbb{E}^{n+m-1}$, то для $u_{0} \in E \cap M_{n+m}, u_{0} \neq 0$, имеем $p\left(u_{0}\right) \leqslant \lambda_{n}$. Следовательно,

$$
\max _{E \in \mathbb{E}^{n+m-1}} \min _{0 \neq u \in E} p(u) \leqslant \lambda_{n},
$$

причем равенство достигается для подпространства $E=\mathscr{H} \ominus M_{n+m-1}$ (если $\left.\lambda_{n-1}=\lambda_{n}\right)$ или $E=\mathscr{H}_{n}^{-}\left(\right.$если $\left.\lambda_{n-1}<\lambda_{n}\right)$.

ТЕОРема 3. Для СЗ $\lambda_{1}, \lambda_{2}, \ldots, \lambda_{n}, \ldots$ ОФ L справедлив вариачионныи приниип Фишера-Куранта-Вейля:

$$
\lambda_{n}=\min _{E \in \mathbb{E}_{n+m}} \max _{0 \neq u \in E} p(u) .
$$

ДокаЗАТЕльство. Если $E \in \mathbb{E}_{n+m}$, то для $u_{0} \in E \cap \mathscr{H}_{n}^{-}, u_{0} \neq 0$, имеем $p\left(u_{0}\right) \geqslant \lambda_{0}$. Следовательно,

$$
\min _{E \in \mathbb{E}_{n+m}} \max _{0 \neq u \in E} p(u) \geqslant \lambda_{n},
$$

причем равенство достигается для подпространства $M_{n+m}$.

Отметим еще следующее простое следствие теоремы 1 :

$$
\begin{aligned}
& N(a, \lambda)=\pi(\lambda)-\pi(a+0) \\
& \quad=\max \{\operatorname{dim} E: E \subset \mathscr{H}, \forall u \in E, u \neq 0 \quad(L(\lambda) u, u)>0 \&(L(a+0) u, u)<0\} \\
& \quad=\max \{\operatorname{dim} E: E \subset \mathscr{H}, \forall u \in E, u \neq 0 \quad a<p(u)<\lambda\} .
\end{aligned}
$$


ЗАмечание 3. В работах [1], [2] аналоги равенств (3) и (4) доказаны при дополнительных предположениях об ограниченности функционала Рэлея или полноте системы СВ ОФ $L(\lambda)$; в работе [3] вариационные принципы (3) и (4) установлены в случае $m=0$ c sup и inf вместо $\max$ и $\min$.

Московский государственный университет

Поступило им. М.В. Ломоносова 30.11 .95

Киевский государственный университет

им. Т. Г. Шевченко

\section{СПИСОК ЦИТИРОВАННОЙ ЛИТЕРАТУРЫ}

1. Абрамов Ю. Ш. Вариационные методы в теории операторных пучков. Спектральная оптимизация. Л.: Изд-во ЛГУ, 1983. 2. Маркус А. С. Введение в спектральную теорию полиномиальных операторных пучков. Кишинев: Штиинца, 1986. 3. Максудов Ф. Г., Гасанов М. Г. // Докл. РАН. 1992. Т. 325. № 5. С. 915-918. 4. Мельник Т. А., Назаров С. А. // Тр. семинара им. И. Г. Петровского. 1996. T. 19. 5. Mel'nyk T. A., Nazarov S. A. // C. R. Acad. Sci. Paris. Sér. 1. 1994. V. 346. P. 1343-1348. 6. Шкаликов А. А., Гринив Р. О. // Матем. заметки. 1994. Т. 56. № 2. С. 114-131. 7. Мельник Т. А. // Доповіді АН України. 1994. № 12 . C. $7-9$. 